\title{
THE VARIETY GENERATED BY AN AI-SEMIRING OF ORDER THREE
}

\author{
Xianzhong Zhao ${ }^{1}$, Miaomiao Ren ${ }^{1, \dagger}$, Siniša Crvenković ${ }^{2}$, \\ Yong Shao ${ }^{1}$, Petar Dapić ${ }^{2}$ \\ ${ }^{1}$ Northwest University, Xi'an, 710069, P.R. China \\ ${ }^{2}$ University of Novi Sad, Novi Sad, 21102, Serbia \\ ${ }^{\dagger}$ miaomiaoren@yeah.net
}

\begin{abstract}
Up to isomorphism, there are 61 ai-semirings of order three. The finite basis problem for these semirings is investigated. This problem for 45 semirings of them is answered by some results in the literature. The remaining semirings are studied using equational logic. It is shown that with the possible exception of the semiring $S_{7}$, all ai-semirings of order three are finitely based.
\end{abstract}

Keywords: Ai-semiring, Identity, Finitely based variety.

\section{Introduction and preliminaries}

By a variety we mean a class of algebras of the same type that is closed under subalgebras, homomorphic images and direct products. It is well-known (Birkhoff's theorem) that a class of algebras of the same type is a variety if and only if it is an equational class. One of the fundamental problems about a variety is the so called finite basis problem, that is, whether it can be defined by finitely many identities. If the answer is positive, then it is called finitely based. Otherwise, it is called nonfinitely based. An algebra $A$ is said to be finitely based (resp., nonfinitely based) if the variety generated by $A$ is finitely based (resp., nonfinitely based).

In 1951 Lyndon [9] showed that all two-element algebras are finitely based and formulated the problem whether every finite algebra is finitely based. This problem has been answered negatively, since a certain seven-element groupoid [10] was shown to be nonfinitely based. Some classical algebras are finite based. For example, so are every finite group [15], every finite associative ring $[6,8]$, every finite lattice [11] and every commutative semigroup [18]. However, not every finite semigroup and not every finite semiring are finitely based. The first example of an nonfinitely based finite semigroup (resp., semiring) has been given by Perkins [18] (resp., Dolinka [1]).

To seek a ultimate solution to the finite basis problem for finite algebras, Tarski [24] proposed the following problem: Is there an algorithm to decide whether a finite algebra is finitely based? McKenzie [12] negatively answered this problem for finite groupoids. However, this problem is still open when restricted to finite semigroups and finite semirings.

By a semiring we mean an algebra $(S,+, \cdot)$ such that

- the additive reduct $(S,+)$ is a commutative semigroup;

- the multiplicative reduct $(S, \cdot)$ is a semigroup;

- $(S,+, \cdot)$ satisfies the identities $x(y+z) \approx x y+x z$ and $(y+z) x \approx y x+z x$.

One can easily find many examples of semirings in almost all branches of mathematics. Semirings can be regarded as a common generalization of both rings and distributive lattices. They have 
been widely applicated in theoretical computer science and information science. We shall say that a semiring is an additively idempotent semiring (ai-semiring for short) if its additive reduct is a semilattice, i.e., a commutative idempotent semigroup. The variety of all ai-semirings is denoted by AI. Let $P_{f}\left(X^{+}\right)$denote the set of all finite non-empty subsets of the free semigroup $X^{+}$on a countably infinite set $X$ of variables. If we define an addition and a multiplication on $P_{f}\left(X^{+}\right)$by

$$
A+B=A \cup B, \quad A \circ B=\{a b \mid a \in A, b \in B\},
$$

then $\left(P_{f}\left(X^{+}\right),+, \circ\right)$ is free in $\mathbf{A I}$ with respect to the mapping $\varphi: X \rightarrow P_{f}\left(X^{+}\right), x \mapsto\{x\}$ (see [7, Theorem 2.5]). An ai-semiring identity (AI-identity for short) over $X$ is an expression of the form $u \approx v$, where $u, v \in P_{f}\left(X^{+}\right)$. For convenience, we write $u_{1}+u_{2}+\cdots+u_{k} \approx v_{1}+v_{2}+\cdots+v_{\ell}$ for the ai-semiring identity $\left\{u_{i} \mid 1 \leq i \leq k\right\} \approx\left\{v_{j} \mid 1 \leq j \leq \ell\right\}$.

In the last decades, several authors studied the finite basis problem for various semiring varieties. There is a rich literature on this subject (see [1-5, 16, 17, 19-23, 25, 27, 28]). Dolinka [1] found the first example of a finite nonfinitely based ai-semiring. In [2] he provided a sufficient condition under which an ai-semiring is inherently nonfinitely based, i.e., the variety $\mathbf{V}$ generated by this semiring is locally finite and every locally finite variety $\mathbf{W}$ for which $\mathbf{V} \subseteq \mathbf{W}$ is nonfinitely based. As an application, it was shown in $[3,4]$ that some ai-semirings are inherently nonfinitely based ${ }^{1}$. McKenzie and Romanowska [13] showed that all ai-semirings satisfying $x^{2} \approx x$ and $x y \approx y x$ are finitely based. Zhao et al. [27, 28] considered the finite basis problem for ai-semirings satisfying $x^{2} \approx x$ that are related to Green's relations. Based on the work of [13, 27, 28], Ghosh et al. [5] and Pastijn [16] proved that all ai-semirings satisfying $x^{2} \approx x$ are finitely based. Ren et al. [21] showed that this result holds for all ai-semirings satisfying $x^{3} \approx x$. However, not every ai-semirings satisfying $x^{n} \approx x(n \geq 4)$ is finitely based (see [22]). Recently, Ren et al. [20] answered the finite basis problem for ai-semirings satisfying $x^{n} \approx x$ and $x y \approx y x$ in which $n-1$ is square-free. From these references one can find that semirings of small order have played an important role. This motivates some authors to investigate the finite basis problem for ai-semirings of small order. In this direction, Shao and Ren [23] considered the variety generated by all ai-semirings of order two. Vechtomov and Petrov [25] studied the variety generated by all semirings of order two whose multiplicative reduct is a semilattice. Moreover, McNulty and Willard [14] initiated the study of the finite basis problem for algebras of order three. The present paper follows this line of investigation. We shall systematically study the finite basis problem for ai-semirings of order three. For this, the following information about ai-semirings of order two in [23] are necessary.

Up to isomorphism, there are exactly 6 ai-semirings of order two, which are listed as $L_{2}, R_{2}$, $M_{2}, D_{2}, N_{2}$ and $T_{2}$ in Table 1 . We assume that the underlying set of each of these semirings is $\{0,1\}$. Their Cayley tables for addition and multiplication are listed in the 2nd and respectively the 3rd columns of Table 1 while the 4th column contains their equational bases.

To present the solution of the word problem for ai-semirings of order two, we need to introduce the following notations. Let $\omega$ be an element of $X^{+}$and $x$ an element of $\omega$. Then

$\diamond c(\omega)$ denotes the content of $\omega$, i.e., the set of all variables occurring in $\omega$.

$\diamond h(\omega)$ denotes the head of $\omega$, i.e., the first variable occurring in $u$.

$\diamond t(\omega)$ denotes the tail of $\omega$, i.e., the last variable occurring in $u$.

$\diamond \ell(\omega)$ denotes the length of $\omega$, i.e., is the number of variables occurring in $u$, where each letter is counted as many times as it occurs in $u$.

$\diamond m(x, \omega)$ denotes the multiplicity of $x$ in $\omega$, i.e., the number of occurrences of $x$ in $w$.

\footnotetext{
${ }^{1}$ The semiring varieties in Dolinka's papers are types of $(2,2,0)$.
} 
Table 1. The 2-element ai-semirings

\begin{tabular}{|c|c|c|c|}
\hline Semiring & Addition & Multiplication & Equational basis \\
\hline \multirow{2}{*}{$L_{2}$} & $\begin{array}{ll}0 & 1\end{array}$ & $\begin{array}{ll}0 & 0\end{array}$ & \multirow{2}{*}{$x y \approx x$} \\
\hline & 11 & 11 & \\
\hline \multirow{2}{*}{$R_{2}$} & 1 & 0 & \multirow{2}{*}{$x y \approx y$} \\
\hline & 11 & 0 & \\
\hline \multirow{2}{*}{$M_{2}$} & 0 & $\begin{array}{ll}0 & 1\end{array}$ & \multirow{2}{*}{$x+y \approx x y$} \\
\hline & 1 & 11 & \\
\hline \multirow{2}{*}{$D_{2}$} & 0 & 0 & \multirow{2}{*}{$x^{2} \approx x, x y \approx y x, x+x y \approx x$} \\
\hline & 1 & 0 & \\
\hline \multirow{2}{*}{$N_{2}$} & 0 & 0 & \multirow{2}{*}{$x y \approx z t, x+x^{2} \approx x$} \\
\hline & 1 & 0 & \\
\hline \multirow[t]{2}{*}{$T_{2}$} & 0 & 1 & \multirow[t]{2}{*}{$x y \approx z t, x+x^{2} \approx x^{2}$} \\
\hline & 1 & 1 & \\
\hline
\end{tabular}

The following result follows from [23, Lemma 1.1]. We shall directly apply it without further notice.

Lemma 1. Let $u \approx v$ be an nontrivial AI-identity, where $u=u_{1}+\cdots+u_{k}, v=v_{1}+\cdots+$ $v_{\ell}, u_{i}, v_{j} \in X^{+}, 1 \leq i \leq k, 1 \leq j \leq \ell$. Then

(i) $L_{2}$ satisfies $u \approx v$ if and only if $\left\{h\left(u_{i}\right) \mid 1 \leq i \leq k\right\}=\left\{h\left(v_{j}\right) \mid 1 \leq j \leq \ell\right\}$;

(ii) $R_{2}$ satisfies $u \approx v$ if and only if $\left\{t\left(u_{i}\right) \mid 1 \leq i \leq k\right\}=\left\{t\left(v_{j}\right) \mid 1 \leq j \leq \ell\right\}$;

(iii) $M_{2}$ satisfies $u \approx v$ if and only if $\bigcup\left\{c\left(u_{i}\right) \mid 1 \leq i \leq k\right\}=\bigcup\left\{c\left(v_{j}\right) \mid 1 \leq j \leq \ell\right\}$;

(iv) $D_{2}$ satisfies $u \approx v$ if and only if $\left(\forall u_{i} \in u\right)\left(\exists v_{j} \in v\right) c\left(v_{j}\right) \subseteq c\left(u_{i}\right)$ and $\left(\forall v_{k} \in v\right)\left(\exists u_{\ell} \in\right.$ $u) c\left(u_{\ell}\right) \subseteq c\left(v_{k}\right)$;

(v) $N_{2}$ satisfies $u \approx v$ if and only if $\left\{u_{i} \in u \mid \ell\left(u_{i}\right)=1\right\}=\left\{v_{j} \in v \mid \ell\left(v_{j}\right)=1\right\}$;

(vi) $T_{2}$ satisfies $u \approx v$ if and only if $\left\{u_{i} \in u \mid \ell\left(u_{i}\right) \geq 2\right\} \neq \emptyset,\left\{v_{j} \in v \mid \ell\left(v_{j}\right) \geq 2\right\} \neq \emptyset$.

Up to isomorphism, there are 61 ai-semirings of order three ${ }^{2}$, which are listed as $S_{i}, 1 \leq i \leq 61$ in Table 2. We assume that the carrier set of each of these semirings is $\{1,2,3\}$. Their Cayley tables for addition and multiplication are listed in Table 2. It is easy to check that there are 24 ai-semirings of order three satisfying $x^{3} \approx x$. By the main results of [21] we have that these semirings are all finitely based. So we only need to study the finite basis problem for the remaining 37 semirings. In fact, some of these semirings are members of the variety which are generated by all ai-semirings of order two. By the the main result of [23] it follows that they are all finitely based. Thus we have

Proposition 1. The following ai-semirings are finitely based: $S_{1}, S_{3}, S_{5}, S_{8}, S_{9}, S_{10}, S_{11}$, $S_{12}, S_{13}, S_{14}, S_{15}, S_{16}, S_{17}, S_{18}, S_{19}, S_{20}, S_{21}, S_{22}, S_{23}, S_{24}, S_{25}, S_{26}, S_{27}, S_{28}, S_{29}, S_{30}, S_{31}$, $S_{32}, S_{33}, S_{34}, S_{35}, S_{36}, S_{37}, S_{38}, S_{39}, S_{40}, S_{41}, S_{42}, S_{43}, S_{48}, S_{49}, S_{50}, S_{51}, S_{52}$ and $S_{61}$.

For an ai-semiring $S, S^{*}$ denotes the (multiplicative) left-right dual of $S$. It is easy to see that if $S$ is finitely based, so is $S^{*}$. Thus, in the remaining we only need to study the finite basis problem for $S_{2}, S_{4}, S_{7}, S_{44}, S_{46}, S_{47}, S_{53}, S_{55}, S_{57}, S_{58}, S_{59}$ and $S_{60}$. The following theorem is our main result.

Theorem 1. With the possible exception of $S_{7}$, all ai-semirings of order three are finitely based.

\footnotetext{
${ }^{2}$ We wrote a program and obtained this result.
} 
Xianzhong Zhao et al.

Table 2. The 3-element ai-semirings

\begin{tabular}{|c|c|c|c|c|c|c|c|c|c|c|c|c|c|}
\hline Semiring & & + & & & $\cdot$ & & Semiring & & + & & & . & \\
\hline \multirow{3}{*}{$S_{1}$} & 1 & 1 & 1 & 1 & 1 & 1 & \multirow{3}{*}{$S_{2}$} & 1 & 1 & 1 & 1 & 1 & 1 \\
\hline & 1 & 2 & 1 & 1 & 1 & 1 & & 1 & 2 & 1 & 1 & 1 & 1 \\
\hline & 1 & 1 & 3 & 1 & 1 & 1 & & 1 & 1 & 3 & 1 & 1 & 2 \\
\hline \multirow{3}{*}{$S_{3}$} & 1 & 1 & 1 & 1 & 1 & 1 & \multirow{3}{*}{$S_{4}$} & 1 & 1 & 1 & 1 & 1 & 1 \\
\hline & 1 & 2 & 1 & 1 & 1 & 1 & & 1 & 2 & 1 & 1 & 1 & 1 \\
\hline & 1 & 1 & 3 & 1 & 1 & 3 & & 1 & 1 & 3 & 1 & 2 & 3 \\
\hline \multirow{3}{*}{$S_{5}$} & 1 & 1 & 1 & 1 & 1 & 1 & \multirow{3}{*}{$S_{6}$} & 1 & 1 & 1 & 1 & 1 & 1 \\
\hline & 1 & 2 & 1 & 1 & 1 & 1 & & 1 & 2 & 1 & 1 & 1 & 2 \\
\hline & 1 & 1 & 3 & 3 & 3 & 3 & & 1 & 1 & 3 & 1 & 1 & 3 \\
\hline \multirow{3}{*}{$S_{7}$} & 1 & 1 & 1 & 1 & 1 & 1 & \multirow{3}{*}{$S_{8}$} & 1 & 1 & 1 & 1 & 1 & 1 \\
\hline & 1 & 2 & 1 & 1 & 1 & 2 & & 1 & 2 & 1 & 1 & 2 & 1 \\
\hline & 1 & 1 & 3 & 1 & 2 & 3 & & 1 & 1 & 3 & 1 & 1 & 3 \\
\hline \multirow{3}{*}{$S_{9}$} & 1 & 1 & 1 & 1 & 1 & 1 & \multirow{3}{*}{$S_{10}$} & 1 & 1 & 1 & 1 & 1 & 1 \\
\hline & 1 & 2 & 1 & 1 & 2 & 1 & & 1 & 2 & 1 & 1 & 2 & 3 \\
\hline & 1 & 1 & 3 & 3 & 3 & 3 & & 1 & 1 & 3 & 1 & 3 & 2 \\
\hline \multirow{3}{*}{$S_{11}$} & 1 & 1 & 1 & 1 & 1 & 1 & \multirow{3}{*}{$S_{12}$} & 1 & 1 & 1 & 1 & 1 & 3 \\
\hline & 1 & 2 & 1 & 2 & 2 & 2 & & 1 & 2 & 1 & 1 & 1 & 3 \\
\hline & 1 & 1 & 3 & 3 & 3 & 3 & & 1 & 1 & 3 & 1 & 1 & 3 \\
\hline \multirow{3}{*}{$S_{13}$} & 1 & 1 & 1 & 1 & 1 & 3 & \multirow{3}{*}{$S_{14}$} & 1 & 1 & 1 & 1 & 1 & 3 \\
\hline & 1 & 2 & 1 & 1 & 1 & 3 & & 1 & 2 & 1 & 1 & 2 & 3 \\
\hline & 1 & 1 & 3 & 3 & 3 & 3 & & 1 & 1 & 3 & 1 & 1 & 3 \\
\hline \multirow{3}{*}{$S_{15}$} & 1 & 1 & 1 & 1 & 1 & 1 & \multirow{3}{*}{$S_{16}$} & 1 & 1 & 1 & 1 & 2 & 3 \\
\hline & 1 & 2 & 1 & 1 & 2 & 1 & & 1 & 2 & 1 & 1 & 2 & 3 \\
\hline & 1 & 1 & 3 & 3 & 3 & 3 & & 1 & 1 & 3 & 1 & 2 & 3 \\
\hline \multirow{3}{*}{$S_{17}$} & 1 & 1 & 1 & 2 & 2 & 2 & \multirow{3}{*}{$S_{18}$} & 1 & 1 & 3 & 1 & 1 & 1 \\
\hline & 1 & 2 & 1 & 2 & 2 & 2 & & 1 & 2 & 3 & 1 & 1 & 1 \\
\hline & 1 & 1 & 3 & 2 & 2 & 2 & & 3 & 3 & 3 & 1 & 1 & 1 \\
\hline \multirow{3}{*}{$S_{19}$} & 1 & 1 & 3 & 1 & 1 & 1 & & 1 & 1 & 3 & 1 & 1 & 1 \\
\hline & 1 & 2 & 3 & 1 & 1 & 1 & $S_{20}$ & 1 & 2 & 3 & 1 & 1 & 1 \\
\hline & 3 & 3 & 3 & 1 & 1 & 3 & & 3 & 3 & 3 & 3 & 3 & 3 \\
\hline & 1 & 1 & 3 & 1 & 1 & 1 & & 1 & 1 & 3 & 1 & 1 & 1 \\
\hline$S_{21}$ & 1 & 2 & 3 & 1 & 2 & 1 & $S_{22}$ & 1 & 2 & 3 & 1 & 2 & 1 \\
\hline & 3 & 3 & 3 & 1 & 1 & 1 & & 3 & 3 & 3 & 1 & 1 & 3 \\
\hline & 1 & 1 & 3 & 1 & 1 & 1 & & 1 & 1 & 3 & 1 & 1 & 1 \\
\hline$S_{23}$ & 1 & 2 & 3 & 1 & 2 & 1 & $S_{24}$ & 1 & 2 & 3 & 2 & 2 & 2 \\
\hline & 3 & 3 & 3 & 3 & 3 & 3 & & 3 & 3 & 3 & 1 & 1 & 1 \\
\hline & 1 & 1 & 3 & 1 & 1 & 1 & & 1 & 1 & 3 & 1 & 1 & 1 \\
\hline$S_{25}$ & 1 & 2 & 3 & 2 & 2 & 2 & $S_{26}$ & 1 & 2 & 3 & 2 & 2 & 2 \\
\hline & 3 & 3 & 3 & 1 & 1 & 3 & & 3 & 3 & 3 & 3 & 3 & 3 \\
\hline & 1 & 1 & 3 & 1 & 1 & 3 & & 1 & 1 & 3 & 1 & 1 & 3 \\
\hline$S_{27}$ & 1 & 2 & 3 & 1 & 1 & 3 & $S_{28}$ & 1 & 2 & 3 & 1 & 1 & 3 \\
\hline & 3 & 3 & 3 & 1 & 1 & 3 & & 3 & 3 & 3 & 3 & 3 & 3 \\
\hline & 1 & 1 & 3 & 1 & 1 & 3 & & 1 & 1 & 3 & 1 & 1 & 3 \\
\hline$S_{29}$ & 1 & 2 & 3 & 1 & 2 & 3 & $S_{30}$ & 1 & 2 & 3 & 1 & 2 & 3 \\
\hline & 3 & 3 & 3 & 1 & 1 & 3 & & 3 & 3 & 3 & 3 & 3 & 3 \\
\hline
\end{tabular}




\begin{tabular}{|c|c|c|c|c|c|c|c|c|c|c|c|c|c|}
\hline \multirow{3}{*}{$S_{31}$} & 1 & 1 & 3 & 1 & 1 & 3 & \multirow{3}{*}{$S_{32}$} & 1 & 1 & 3 & 1 & 2 & 1 \\
\hline & 1 & 2 & 3 & 2 & 2 & 3 & & 1 & 2 & 3 & 1 & 2 & 1 \\
\hline & 3 & 3 & 3 & 3 & 3 & 3 & & 3 & 3 & 3 & 1 & 2 & 1 \\
\hline \multirow{3}{*}{$S_{33}$} & 1 & 1 & 3 & 1 & 2 & 1 & \multirow{3}{*}{$S_{34}$} & 1 & 1 & 3 & 1 & 2 & 1 \\
\hline & 1 & 2 & 3 & 1 & 2 & 1 & & 1 & 2 & 3 & 2 & 2 & 2 \\
\hline & 3 & 3 & 3 & 1 & 2 & 3 & & 3 & 3 & 3 & 1 & 2 & 1 \\
\hline \multirow{3}{*}{$S_{35}$} & 1 & 1 & 3 & 1 & 2 & 1 & \multirow{3}{*}{$S_{36}$} & 1 & 1 & 3 & 1 & 2 & 1 \\
\hline & 1 & 2 & 3 & 2 & 2 & 2 & & 1 & 2 & 3 & 2 & 2 & 2 \\
\hline & 3 & 3 & 3 & 1 & 2 & 3 & & 3 & 3 & 3 & 3 & 2 & 3 \\
\hline \multirow{3}{*}{$S_{37}$} & 1 & 1 & 3 & 1 & 2 & 3 & \multirow{3}{*}{$S_{38}$} & 1 & 1 & 3 & 1 & 2 & 3 \\
\hline & 1 & 2 & 3 & 1 & 2 & 3 & & 1 & 2 & 3 & 1 & 2 & 3 \\
\hline & 3 & 3 & 3 & 1 & 2 & 3 & & 3 & 3 & 3 & 3 & 3 & 3 \\
\hline \multirow{3}{*}{$S_{39}$} & 1 & 1 & 3 & 1 & 2 & 3 & \multirow{3}{*}{$S_{40}$} & 1 & 1 & 3 & 1 & 2 & 3 \\
\hline & 1 & 2 & 3 & 2 & 2 & 2 & & 1 & 2 & 3 & 2 & 2 & 2 \\
\hline & 3 & 3 & 3 & 1 & 2 & 3 & & 3 & 3 & 3 & 3 & 2 & 3 \\
\hline \multirow{3}{*}{$S_{41}$} & 1 & 1 & 3 & 1 & 2 & 3 & \multirow{3}{*}{$S_{42}$} & 1 & 1 & 3 & 1 & 2 & 3 \\
\hline & 1 & 2 & 3 & 2 & 2 & 2 & & 1 & 2 & 3 & 2 & 2 & 3 \\
\hline & 3 & 3 & 3 & 3 & 3 & 3 & & 3 & 3 & 3 & 3 & 2 & 3 \\
\hline \multirow{3}{*}{$S_{43}$} & 1 & 1 & 3 & 1 & 2 & 3 & \multirow{3}{*}{$S_{44}$} & 1 & 1 & 3 & 2 & 2 & $\overline{1}$ \\
\hline & 1 & 2 & 3 & 2 & 2 & 3 & & 1 & 2 & 3 & 2 & 2 & 2 \\
\hline & 3 & 3 & 3 & 3 & 3 & 3 & & 3 & 3 & 3 & 1 & 2 & 3 \\
\hline \multirow{3}{*}{$S_{45}$} & 1 & 1 & 3 & 2 & 2 & 1 & \multirow{3}{*}{$S_{46}$} & 1 & 1 & 3 & 2 & 2 & 2 \\
\hline & 1 & 2 & 3 & 2 & 2 & 2 & & 1 & 2 & 3 & 2 & 2 & 2 \\
\hline & 3 & 3 & 3 & 2 & 2 & 3 & & 3 & 3 & 3 & 1 & 2 & 3 \\
\hline \multirow{3}{*}{$S_{47}$} & 1 & 1 & 3 & 2 & 2 & 2 & \multirow{3}{*}{$S_{48}$} & 1 & 1 & 3 & 2 & 2 & 2 \\
\hline & 1 & 2 & 3 & 2 & 2 & 2 & & 1 & 2 & 3 & 2 & 2 & 2 \\
\hline & 3 & 3 & 3 & 2 & 2 & 1 & & 3 & 3 & 3 & 2 & 2 & 2 \\
\hline & 1 & 1 & 3 & 2 & 2 & 2 & & 1 & 1 & 3 & 2 & 2 & 2 \\
\hline$S_{49}$ & 1 & 2 & 3 & 2 & 2 & 2 & $S_{50}$ & 1 & 2 & 3 & 2 & 2 & 2 \\
\hline & 3 & 3 & 3 & 2 & 2 & 3 & & 3 & 3 & 3 & 3 & 3 & 3 \\
\hline & 1 & 1 & 3 & 2 & 2 & 3 & & 1 & 1 & 3 & 2 & 2 & 3 \\
\hline$S_{51}$ & 1 & 2 & 3 & 2 & 2 & 3 & $S_{52}$ & 1 & 2 & 3 & 2 & 2 & 3 \\
\hline & 3 & 3 & 3 & 2 & 2 & 3 & & 3 & 3 & 3 & 3 & 3 & 3 \\
\hline & 1 & 1 & 3 & 3 & 1 & 3 & & 1 & 1 & 3 & 3 & 1 & 3 \\
\hline$S_{53}$ & 1 & 2 & 3 & 1 & 2 & 3 & $S_{54}$ & 1 & 2 & 3 & 3 & 2 & 3 \\
\hline & 3 & 3 & 3 & 3 & 3 & 3 & & 3 & 3 & 3 & 3 & 3 & 3 \\
\hline & 1 & 1 & 3 & 3 & 2 & 3 & & 1 & 1 & 3 & 3 & 2 & 3 \\
\hline$S_{55}$ & 1 & 2 & 3 & 2 & 2 & 2 & $S_{56}$ & 1 & 2 & 3 & 3 & 2 & 3 \\
\hline & 3 & 3 & 3 & 3 & 2 & 3 & & 3 & 3 & 3 & 3 & 2 & 3 \\
\hline & 1 & 1 & 3 & 3 & 3 & 3 & & 1 & 1 & 3 & 3 & 3 & 3 \\
\hline$S_{57}$ & 1 & 2 & 3 & 1 & 2 & 3 & $S_{58}$ & 1 & 2 & 3 & 2 & 2 & 2 \\
\hline & 3 & 3 & 3 & 3 & 3 & 3 & & 3 & 3 & 3 & 3 & 3 & 3 \\
\hline & 1 & 1 & 3 & 3 & 3 & 3 & & 1 & 1 & 3 & 3 & 3 & 3 \\
\hline$S_{59}$ & 1 & 2 & 3 & 3 & 1 & 3 & $S_{60}$ & 1 & 2 & 3 & 3 & 2 & 3 \\
\hline & 3 & 3 & 3 & 3 & 3 & 3 & & 3 & 3 & 3 & 3 & 3 & 3 \\
\hline & 1 & 1 & 3 & 3 & 3 & 3 & & & & & & & \\
\hline$S_{61}$ & 1 & 2 & 3 & 3 & 3 & 3 & & & & & & & \\
\hline & 3 & 3 & 3 & 3 & 3 & 3 & & & & & & & \\
\hline
\end{tabular}




\section{The proof of Theorem 1}

In this section we shall provide the proof of Theorem 1. Let $\operatorname{HSP}(S)$ denote the variety generated by an ai-semiring $S$ and $\underline{k}$ the set $\{1,2, \ldots, k\}$ for a positive integer $k$. We start with a technique that will be used repeatedly in the sequel. Suppose that $\Sigma$ is a set of identities which include the identities that determine $\mathbf{A I}$ and that $u \approx v$ is an AI-identity, where $u=$ $u_{1}+\cdots+u_{k}, v=v_{1}+\cdots+v_{\ell}, u_{i}, v_{j} \in X^{+}, i \in \underline{k}, j \in \underline{\ell}$. Then it is easy to see that the aisemring variety defined by $u \approx v$ is equal to the ai-semiring variety defined by the simpler identities $u \approx u+v_{j}, v \approx v+u_{i}, i \in \underline{k}, j \in \underline{\ell}$. Thus, to show that $u \approx v$ is derivable from $\Sigma$, we only need to show that $u \approx u+v_{j}, v \approx v+u_{i}, i \in \underline{k}, j \in \underline{\ell}$ can be derived from $\Sigma$.

Proposition 2. $\operatorname{HSP}\left(S_{2}\right)$ is the ai-semiring variety determined by the identities

$$
\begin{gathered}
x_{1} x_{2} x_{3} \approx y_{1} y_{2} y_{3}, \\
x+x^{2} \approx x^{3}, \\
x^{2}+y^{2} \approx x y, \\
x^{3}+y \approx x^{3} .
\end{gathered}
$$

P r o o f. An AI-term is said to be in canonical form if it is equal to one of the following terms: $x_{1}+\cdots+x_{m}, x_{1}^{2}+\cdots+x_{m}^{2}, x_{1}+\cdots+x_{m}+y_{1}^{2}+\cdots+y_{n}^{2}$ and $x^{3}$, where $x_{1}, \ldots, x_{m}$ are distinct variables, $y_{1}, \ldots, y_{n}$ are distinct variables, and $\left\{x_{i} \mid i \in \underline{m}\right\} \bigcap\left\{y_{j} \mid j \in \underline{n}\right\}=\emptyset$. Suppose that $u=u_{1}+u_{2}+\cdots+u_{k}$ is an AI-term, where $u_{i} \in X^{+}, i \in \underline{k}$. We shall show that there exists an AI-term $u^{\prime}$ in canonical form such that the identities (1.1)-(1.4) and the identities determining AI imply the identity $u \approx u^{\prime}$. The following cases are needed.

- $\ell\left(u_{i}\right)=1$ for all $i \in \underline{k}$. Then $u=x_{1}+\cdots+x_{m}$.

- $\ell\left(u_{i}\right)=2$ for all $i \in \underline{k}$. Then the identity (1.3) implies $u \approx x_{1}^{2}+\cdots+x_{m}^{2}$.

- $\ell\left(u_{i}\right) \leq 2$ for all $i \in \underline{k}, \ell\left(u_{i_{1}}\right)=1$ for some $i_{1} \in \underline{k}$ and $\ell\left(u_{i_{2}}\right)=2$ for some $i_{2} \in \underline{k}$. If $c\left(u_{i}\right) \bigcap c\left(u_{j}\right) \neq \emptyset$ for some $u_{i}$ and $u_{j}$ with $\ell\left(u_{i}\right)=1$ and $\ell\left(u_{j}\right)=2$, then the identities (1.2)(1.4) implies $u \approx x^{3}$. Otherwise, we have that the identity (1.3) implies $u \approx x_{1}+\cdots+x_{m}+$ $y_{1}^{2}+\cdots+y_{n}^{2}$, where $\left\{x_{i} \mid i \in \underline{m}\right\} \bigcap\left\{y_{j} \mid j \in \underline{n}\right\}=\emptyset$.

- $\ell\left(u_{i}\right) \geq 3$ for some $i \in \underline{k}$. Then the identities (1.1) and (1.4) imply $u \approx x^{3}$.

It is routine to check that $S_{2}$ satisfies the identities (1.1)-(1.4). In the remainder we shall show that every identity which is satisfied in $S_{2}$ can be derived from the identities (1.1)-(1.4) and the identities determining AI. By the above arguments it is enough to show that if $S_{2}$ satisfies an identity $u \approx v$, where $u$ and $v$ are AI-terms in canonical forms, then the identities (1.1)-(1.4) and the identities determining $\mathbf{A I}$ imply $u \approx v$. Notice that $T_{2}$ can be embedded into $S_{2}$. We only need to consider the following cases:

- $u=x_{1}+\cdots+x_{m}, v=y_{1}+\cdots+y_{n}$. It is easy to see that $u \approx v$ is trivial.

- $u=x_{1}^{2}+\cdots+x_{m}^{2}, v=y_{1}^{2}+\cdots+y_{n}^{2}$. It is easy to see that $u \approx v$ is trivial.

- $u=x_{1}^{2}+\cdots+x_{m}^{2}, v=y_{1}+\cdots+y_{k}+z_{1}^{2}+\cdots+z_{\ell}^{2}$. Let $\varphi: P_{f}\left(X^{+}\right) \rightarrow S_{2}$ be a semiring homomorphism such that $\varphi(x)=3$ for every variable $x$ in $X$. Then $\varphi(u)=2$ and $\varphi(v)=1$, a contradiction. Thus $u \approx v$ is not satisfied in $S_{2}$.

- $u=x_{1}^{2}+\cdots+x_{m}^{2}, v=t^{3}$. This case is similar to the preceding one. 
- $u=y_{1}+\cdots+y_{k}+z_{1}^{2}+\cdots+z_{\ell}^{2}, v=y_{1}^{\prime}+\cdots+y_{m}^{\prime}+z_{1}^{\prime 2}+\cdots+z_{n}^{\prime 2}$. It is easy to see that $u \approx v$ is trivial.

- $u=y_{1}+\cdots+y_{k}+z_{1}^{2}+\cdots+z_{\ell}^{2}, v=x^{3}$. Let $\varphi: P_{f}\left(X^{+}\right) \rightarrow S_{2}$ be a semiring homomorphism such that $\varphi\left(y_{i}\right)=2, \varphi\left(z_{j}\right)=3$ and $\varphi(t)=1$ for all $i \in \underline{k}, j \in \underline{\ell}$. Then $\varphi(u)=2$ and $\varphi(v)=1$, a contradiction. Thus $u \approx v$ is not satisfied in $S_{2}$.

- $u=x_{1}^{3}, v=x_{2}^{3}$. Then the identity (1) implies $u \approx v$.

This completes the proof.

Proposition 3. $\operatorname{HSP}\left(S_{4}\right)$ is the ai-semiring variety determined by the identities

$$
\begin{gathered}
x y \approx x^{2} y, \\
x y z \approx y x z, \\
x+y^{2} \approx x y^{2}, \\
x+y z \approx y x+y z .
\end{gathered}
$$

P r o o f. An AI-term is said to be in canonical form if it is equal to one of the following terms: $x_{1}+\cdots+x_{m}, x_{1}^{2} \cdots x_{m}^{2}$ and $x_{1}^{2} \cdots x_{m}^{2}\left(y_{1}+\cdots+y_{n}\right)$, where $x_{1}, \ldots, x_{m}$ are distinct variables, $y_{1}, \ldots, y_{n}$ are distinct variables and $\left\{x_{i} \mid i \in \underline{m}\right\} \bigcap\left\{y_{j} \mid j \in \underline{n}\right\}=\emptyset$. Suppose that $u=u_{1}+u_{2}+\cdots+u_{k}$ is an AI-term, where $u_{i} \in X^{+}, i \in \underline{k}$. We shall show that there exists an AI-term $u^{\prime}$ in canonical form such that the identities (1.5)-(1.8) and the identities determining AI imply the identity $u \approx u^{\prime}$. The following cases are needed.

- $\ell\left(u_{i}\right)=1$ for all $i \in \underline{k}$. Then $u=x_{1}+\cdots+x_{m}$.

- $m\left(t\left(u_{i}\right), u_{i}\right) \geq 2$ for some $j \in \underline{k}$. Then the identities (1.5)-(1.7) imply $u \approx x_{1}^{2} \cdots x_{m}^{2}$.

- $\ell\left(u_{i}\right) \geq 2$ for some $i \in \underline{k}, m\left(t\left(u_{j}\right), u_{j}\right)=1$ for every $j \in \underline{k}$. Then the identities (1.5), (1.6) and (1.8) imply $u \approx x_{1}^{2} \cdots x_{m}^{2}\left(y_{1}+\cdots+y_{n}\right)$, where $\left\{x_{i} \mid i \in \underline{m}\right\} \bigcap\left\{y_{j} \mid j \in \underline{n}\right\}=\emptyset$.

It is routine to check that $S_{4}$ satisfies the identities (1.5)-(1.8). By the above arguments it is enough to show that if $S_{4}$ satisfies an identity $u \approx v$, where $u$ and $v$ are AI-terms in canonical forms, then the identities (1.5)-(1.8) and the identities determining $\mathbf{A I}$ imply $u \approx v$. Since $T_{2}$ can be embedded into $S_{4}$, we only need to consider the following cases:

- $u=x_{1}+\cdots+x_{m}, v=y_{1}+\cdots+y_{n}$. It is easy to see that $u \approx v$ is trivial.

- $u=x_{1}^{2} \cdots x_{m}^{2}, v=y_{1}^{2} \cdots y_{n}^{2}$. It is easy to see that $u \approx v$ is trivial.

- $u=x_{1}^{2} \cdots x_{m}^{2}, v=y_{1}^{2} \cdots y_{k}^{2}\left(z_{1}+\cdots+z_{\ell}\right)$. Let $\varphi: P_{f}\left(X^{+}\right) \rightarrow S_{4}$ be a semiring homomorphism such that $\varphi\left(y_{i}\right)=3, \varphi\left(z_{j}\right)=2, i \in \underline{k}, j \in \underline{\ell}, \varphi(x)=1$ for every remaining variable $x$. Then $\varphi(u)=1$ or $3, \varphi(v)=2$, a contradiction. Thus $u \approx v$ is not satisfied in $S_{4}$.

- $u=x_{1}^{2} \cdots x_{m}^{2}\left(y_{1}+\cdots+y_{n}\right), v=z_{1}^{2} \cdots z_{k}^{2}\left(t_{1}+\cdots+t_{\ell}\right)$. It is easy to see that $u \approx v$ is trivial.

This completes the proof.

Proposition 4. $\mathbf{H S P}\left(S_{44}\right)$ is the ai-semiring variety determined by the identities

$$
\begin{gathered}
x^{3} \approx x^{2}, \\
x y \approx y x, \\
x+x y \approx x, \\
x^{2} y+x y^{2} \approx x y .
\end{gathered}
$$


P r o o f. An AI-term $u=u_{1}+\cdots+u_{n}$ is said to be in canonical form if every $u_{i}$ is equal to one of the following terms: $x, x_{1}^{2} \cdots x_{m}^{2}$ and $x_{1}^{2} \cdots x_{m}^{2} y$, where $x_{1}, \ldots, x_{m}$ are distinct variables and $y \neq x_{i}$ for every $i \in \underline{m}$. Let $p=x_{1} \cdots x_{n}$ be an element of $X^{+}$such that $n \geq 2$. By induction on $n$ we have that the identity (1.10) and (1.12) imply $p \approx \sum_{1 \leq i \leq n} x_{1}^{2} \cdots x_{i-1}^{2} x_{i+1}^{2} \cdots x_{n}^{2} x_{i}$. It follows that for any AI-term $u$, there exists an AI-term $u^{\prime}$ in canonical form such that (1.9)-(1.12) imply $u \approx u^{\prime}$.

It is easy to check that $S_{44}$ satisfies the identities (1.9)-(1.12). To show that $\operatorname{HSP}\left(S_{44}\right)$ is determined by (1.9)-(1.12), by the above arguments it suffices to show that if $S_{44}$ satisfies $u+p \approx u$, where $u+p$ and $u$ are AI-terms in canonical forms, then the identities (1.9)-(1.12) and the identities determining $\mathbf{A I}$ imply $u+p \approx u$. We shall consider the following three cases.

- $p=x$. Since $N_{2}$ can be embedded into $S_{44}$, there exists some $u_{i}$ in $u$ such that $u_{i}=x$. It follows that $u+p \approx u$ is trivial.

- $p=x_{1}^{2} \cdots x_{m}^{2}$. Since $D_{2}$ can be embedded into $S_{44}$, there exists some $u_{i}$ in $u$ such that $c\left(u_{i}\right) \subseteq c(p)$ and so (1.9) and (1.10) imply $u_{i} p \approx p$. Further, we have

$$
u \approx u+u_{i} \stackrel{(1.11)}{\approx} u+u_{i}+u_{i} p \approx u+u_{i}+p \approx u+p .
$$

- $p=x_{1}^{2} \cdots x_{m}^{2} y$. Since $D_{2}$ can be embedded into $S_{44}$, we have that $\left\{u_{i} \in u \mid c\left(u_{i}\right) \subseteq c(p)\right\}$ is non-empty. Suppose that for any $u_{i}$ in $\left\{u_{i} \in u \mid c\left(u_{i}\right) \subseteq c(p)\right\}$, there exists $x$ in $c\left(u_{i}\right)$ such that $m(x, p)<m\left(x, u_{i}\right)$. That is to say, $m\left(y, u_{i}\right)=2$ for every $u_{i}$ in $\left\{u_{i} \mid c\left(u_{i}\right) \subseteq c(p)\right\}$. Let $\varphi: P_{f}\left(X^{+}\right) \rightarrow S_{44}$ be a semiring homomorphism such that $\varphi(z)=2$ for every $z \in X \backslash c(p)$, $\varphi\left(x_{i}\right)=3$ for every $i \in \underline{m}$ and $\varphi(y)=1$. Then $\varphi(u)=2$ and $\varphi(u+p)=1$, a contradiction. Thus there exists $u_{i}$ in $\left\{u_{i} \in u \mid c\left(u_{i}\right) \subseteq c(p)\right\}$ such that $m\left(x, u_{i}\right) \leq m(x, p)$ for every $x$ in $c\left(u_{i}\right)$. If $y \in c\left(u_{i}\right)$, then $m\left(y, u_{i}\right)=1$ and so (1.9) and (1.10) imply $u_{i} x_{1}^{2} \cdots x_{m}^{2} \approx p$. Further, we have

$$
u \approx u+u_{i} \stackrel{(1.11)}{\approx} u+u_{i}+u_{i} x_{1}^{2} \cdots x_{m}^{2} \approx u+p .
$$

If $y \notin c\left(u_{i}\right)$, then (1.9) and (1.10) imply $u_{i} p \approx p$. We therefore have

$$
u \approx u+u_{i} \stackrel{(1.11)}{\approx} u+u_{i}+u_{i} p \approx u+p .
$$

This completes the proof.

Proposition 5. $\mathbf{H S P}\left(S_{46}\right)$ is the ai-semiring variety determined by the identities

$$
\begin{gathered}
x^{2} y \approx x y, \\
x^{2} y^{2} \approx y^{2} x^{2}, \\
x y z \approx y x z, \\
x+x y \approx x, \\
x+y x \approx x .
\end{gathered}
$$

P r o o f. An AI-term $u=u_{1}+\cdots+u_{n}$ is said to be in canonical form if every $u_{i}$ is equal to one of the following terms: $x, x_{1}^{2} \cdots x_{m}^{2}$ and $x_{1}^{2} \cdots x_{m}^{2} y$, where $y \neq x_{i}$ for all $i \in \underline{m}$. Let $p$ be an element of $X^{+}$such that $\ell(p) \geq 2$. If $m(t(p), p)=1$, then the identities (1.13)-(1.15) imply $p \approx x_{1}^{2} \cdots x_{m}^{2} y$. If $m(t(p), p) \geq 2$, then the identities (1.13)-(1.15) imply $p \approx x_{1}^{2} \cdots x_{m}^{2}$. It follows that for any AI-term $u$, there exists an AI-term $u^{\prime}$ in canonical form such that (1.13)-(1.15) imply $u \approx u^{\prime}$. 
It is routine to check that $S_{46}$ satisfies the identities (1.13)-(1.17). To show that $\mathbf{H S P}\left(S_{46}\right)$ is the ai-semiring variety determined by (1.13)-(1.17), by the above arguments it suffices to show that if $S_{46}$ satisfies $u+p \approx u$, where $u+p$ and $u$ are AI-terms in canonical form, then (1.13)-(1.17) imply $u+p \approx u$. The following three cases are necessary.

- $p=x$. Since $D_{2}$ can be embedded into $S_{46}$, there exists some $u_{i}$ in $u$ such that $c\left(u_{i}\right)=\{x\}$. Suppose that $u_{i}=x^{2}$ for every $u_{i}$ in $u$ with $c\left(u_{i}\right)=\{x\}$. Let $\varphi: X \rightarrow S_{46}$ be a semiring homomorphism such that $\varphi(x)=1$ and $\varphi(y)=2$ for every $y \neq x$. Then $\varphi(u)=2$ and $\varphi(u+p)=1$, a contradiction. Thus there exists $u_{i}$ in $u$ such that $u_{i}=x$ and so $u+p \approx u$ is trivial.

- $p=x_{1}^{2} \cdots x_{m}^{2}$. Since $D_{2}$ can be embedded into $S_{46}$, there exists some $u_{i}$ in $u$ such that $c\left(u_{i}\right) \subseteq c(p)$ and so (1.13)-(1.15) imply $p \approx u_{i} p$. We now have

$$
u+p \approx u+u_{i}+p \approx u+u_{i}+u_{i} p \stackrel{(1.16)}{\approx} u+u_{i} \approx u .
$$

- $p=x_{1}^{2} \cdots x_{m}^{2} y$. Since $D_{2}$ can be embedded into $S_{46}$, it follows that $\left\{u_{i} \in u \mid c\left(u_{i}\right) \subseteq c(p)\right\}$ is non-empty. Suppose that $m\left(y, u_{i}\right)=2$ for every $u_{i}$ in $\left\{u_{i} \in u \mid c\left(u_{i}\right) \subseteq c(p)\right\}$. Let $\varphi: P_{f}\left(X^{+}\right) \rightarrow S_{46}$ be a semiring homomorphism such that $\varphi(z)=2$ for every $z \notin c(p)$, $\varphi\left(x_{i}\right)=3$ for every $i \in \underline{m}$ and $\varphi(y)=1$. Then $\varphi(u)=2$ and $\varphi(u+p)=1$, a contradiction. Thus we only need to consider the following cases:

$\diamond\left(\exists u_{i} \in\left\{u_{i} \in u \mid c\left(u_{i}\right) \subseteq c(p)\right\}\right) y \notin c\left(u_{i}\right)$. Then

$$
u+p \approx u+u_{i}+p \stackrel{(1.13) \underset{\sim}{\approx}(1.15)}{\approx} u+u_{i}+u_{i} p \stackrel{(1.16)}{\approx} u+u_{i} \approx u .
$$

$\diamond\left(\exists u_{i} \in\left\{u_{i} \in u \mid c\left(u_{i}\right) \subseteq c(p)\right\}\right) y \in c\left(u_{i}\right), t\left(u_{i}\right)=y$ and $m\left(y, u_{i}\right)=1$. Then

$$
u+p \approx u+u_{i}+p \stackrel{(1.13)-(1.15)}{\approx} u+u_{i}+x_{1}^{2} \cdots x_{m}^{2} u_{i} \stackrel{(1.17)}{\approx} u+u_{i} \approx u .
$$

This completes the proof.

Proposition 6. $\operatorname{HSP}\left(S_{47}\right)$ is the ai-semiring variety determined by the identities

$$
\begin{gathered}
x y \approx y x, \\
x+x y \approx x, \\
x^{2}+x y \approx x^{2}, \\
x+x_{1} x_{2} x_{3} \approx x .
\end{gathered}
$$

P r o o f. It is easy to verify that $S_{47}$ satisfies the identities (1.18)-(1.21). In the remainder it suffices to show that every identity which is satisfied in $S_{47}$ is derivable from (1.18)-(1.21). Let $u+p \approx u$ be such an identity, where $u=u_{1}+\cdots+u_{m}, u_{i}, p \in X^{+}, i \in \underline{m}$. We consider the following three cases.

- $\ell(p)=1$. Since $N_{2}$ can be embedded into $S_{47}$, there exists $u_{i}$ in $u$ such that $u_{i}=p$. Thus $u+p \approx u$ is trivial. 
- $\ell(p)=2$. Suppose that for any $u_{i}$ in $u, c\left(u_{i}\right) \nsubseteq c(p)$. Let $\varphi: P_{f}\left(X^{+}\right) \rightarrow S_{47}$ be a semiring homomorphism such that $\varphi(z)=2$ for every $z \in X \backslash c(p)$ and $\varphi(x)=3$ for every $x \in c(p)$. Then $\varphi(u)=2$ and $\varphi(u+p)=1$, a contradiction. Thus there exists $u_{i}$ in $u$ such that $c\left(u_{i}\right) \subseteq c(p)$. Assume that $\ell\left(u_{i}\right) \geq 3$ for every $u_{i}$ in $\left\{u_{i} \in u \mid c\left(u_{i}\right) \subseteq c(p)\right\}$. Then $\varphi(u)=2$ and $\varphi(u+p)=1$, a contradiction. This implies that there exists $u_{i}$ in $u$ such that $c\left(u_{i}\right) \subseteq c(p)$ and $\ell\left(u_{i}\right) \leq 2$. Further, the identities (1.18)-(1.20) imply

$$
u+p \approx u+u_{i}+p \approx u+u_{i} \approx u .
$$

- $\ell(p) \geq 3$. Then $u+p \approx u$ can be derived from (1.21).

This completes the proof.

Proposition 7. $\mathbf{H S P}\left(S_{53}\right)$ is the ai-semiring variety determined by the identities

$$
\begin{gathered}
x y \approx y x, \\
x y+y^{2} \approx x+y^{2}, \\
x+x y \approx x y, \\
x y+y z+x z \approx x y z .
\end{gathered}
$$

P r o o f. An AI-term is said to be in canonical form if it is equal to one of the following terms: $x_{1}+\cdots+x_{m}, x_{1} y_{1}+\cdots+x_{m} y_{m}$, and $x_{1}+\cdots+x_{m}+y_{1} z_{1}+\cdots+y_{n} z_{n}$, where $\left\{x_{i} \mid i \in \underline{m}\right\} \bigcap\left\{y_{j}, z_{j} \mid\right.$ $j \in \underline{n}\}=\emptyset, x^{2}$ and $x y$ can not occur simultaneously. Suppose that $u=u_{1}+u_{2}+\cdots+u_{k}$ is an AI-term, where $u_{i} \in X^{+}, i \in \underline{k}$. It is easy to show that there exists an AI-term $u^{\prime}$ in canonical form such that the identities (1.22)-(1.25) imply the identity $u \approx u^{\prime}$.

It is routine to check that $S_{53}$ satisfies the identities (1.22)-(1.25). In the remainder we shall show that every identity which is satisfied in $S_{53}$ can be derived from the identities (1.22)-(1.25). By the above arguments it suffices to show that if $S_{53}$ satisfies an identity $u \approx v$, where $u$ and $v$ are AI-terms in canonical form, then the identities (1.22)-(1.25) and the identities determining $\mathbf{A I}$ imply $u \approx v$. Notice that $T_{2}$ can be embedded into $S_{53}$. We only need to consider the following cases:

- $u=x_{1}+\cdots+x_{m}, v=y_{1}+\cdots+y_{n}$. It follows immediately that $u \approx v$ is trivial.

- $u=x_{1} y_{1}+\cdots+x_{m} y_{m}, v=z_{1} s_{1}+\cdots+z_{n} s_{n}$. For any $i \in \underline{m}$, suppose that $\left\{x_{1}, y_{1}\right\}$ is not equal to $\left\{z_{j}, s_{j}\right\}$ for every $j \in \underline{n}$. Consider the following two subcases:

$\diamond x_{i}=y_{i}$. Let $\varphi: P_{f}\left(X^{+}\right) \rightarrow S_{53}$ be a semiring homomorphism such that $\varphi\left(x_{i}\right)=1$ and $\varphi(z)=2$ for every $z \in X \backslash\left\{x_{i}\right\}$. Then $\varphi(u)=3$ and $\varphi(v)=1$, a contradiction.

$\diamond x_{i} \neq y_{i}$. Since $M_{2}$ can be embedded into $S_{53}$, we can deduce that $S_{53}$ satisfies one of the following identities: $x_{i} y_{i} \approx x_{i}^{2}+y_{i}^{2}, x_{i} y_{i} \approx x_{i}^{2}+y_{i}, x_{i} y_{i} \approx x_{i}+y_{i}^{2}$ and $x_{i} y_{i} \approx x_{i}+y_{i}$, a contradiction.

Thus $\left\{x_{i}, y_{i}\right\}$ is equal to $\left\{z_{j}, s_{j}\right\}$ for some $j \in \underline{n}$. Similarly, for any $j \in \underline{n},\left\{z_{j}, s_{j}\right\}$ is equal to $\left\{x_{i}, y_{i}\right\}$ for some $i \in \underline{m}$. Hence $u \approx v$ is trivial.

- $u=x_{1} y_{1}+\cdots+x_{m} y_{m}, v=z_{1}+\cdots+z_{k}+s_{1} t_{1}+\cdots+s_{\ell} t_{\ell}$. We consider the following two subcases.

$\diamond\left\{x_{i}, y_{i}\right\} \subseteq\left\{z_{i} \mid i \in \underline{k}\right\}$ for some $i \in \underline{m}$. Let $\psi: P_{f}\left(X^{+}\right) \rightarrow S_{53}$ be a semiring homomorphism such that $\psi\left(z_{i}\right)=1$ for every $i \in \underline{k}$ and $\psi(x)=2$ for every $z \in X \backslash\left\{z_{i} \mid i \in \underline{k}\right\}$. Then $\psi(u)=3$ and $\psi(v)=1$, a contradiction. 
$\diamond\left\{x_{i}, y_{i}\right\} \nsubseteq\left\{z_{i} \mid i \in \underline{k}\right\}$ for every $i \in \underline{m}$. Notice that $M_{2}$ can be embedded into $S_{53}$. Let $\theta: P_{f}\left(X^{+}\right) \rightarrow S_{53}$ be a semiring homomorphism such that $\theta\left(x_{i}\right)=\theta\left(y_{i}\right)=1$ if $\left\{x_{i}, y_{i}\right\} \bigcap\left\{z_{i} \mid i \in \underline{k}\right\} \neq \emptyset$, and $\theta(y)=2$ for every remaining variable $y$. Then $\theta(u)=3$ and $\theta(v)=1$, a contradiction.

This shows that $u \approx v$ is not satisfied in $S_{53}$.

- $u=x_{1}+\cdots+x_{m}+y_{1} z_{1}+\cdots+y_{n} z_{n}, v=x_{1}^{\prime}+\cdots+x_{k}^{\prime}+y_{1}^{\prime} z_{1}^{\prime}+\cdots+y_{\ell}^{\prime} z_{\ell}^{\prime}$. Suppose that $x_{1}=y_{i}^{\prime}$ for some $i$.

$\diamond y_{i}^{\prime}=z_{i}^{\prime}$. Choose every variable in $X \backslash\left\{x_{1}\right\}$ to 2 . Then $S_{53}$ satisfies $x_{1} \approx x_{1}^{2}$, a contradiction.

$\diamond y_{i}^{\prime} \neq z_{i}^{\prime}$. Choose every variable in $X \backslash\left\{y_{i}^{\prime}, z_{i}^{\prime}\right\}$ to 2 . Then $S_{53}$ satisfies $y_{i}^{\prime}+z_{i}^{\prime} \approx y_{i}^{\prime} z_{i}^{\prime}$ or $y_{i}^{\prime}+z_{i}^{\prime 2} \approx y_{i}^{\prime} z_{i}^{\prime}$, a contradiction.

This implies that $x_{1}+\cdots+x_{m} \approx x_{1}^{\prime}+\cdots+x_{k}^{\prime}$ is trivial and so $S_{53}$ satisfies $y_{1} z_{1}+\cdots+y_{n} z_{n} \approx$ $y_{1}^{\prime} z_{1}^{\prime}+\cdots+y_{\ell}^{\prime} z_{\ell}^{\prime}$. By the preceding case it follows that $y_{1} z_{1}+\cdots+y_{n} z_{n} \approx y_{1}^{\prime} z_{1}^{\prime}+\cdots+y_{\ell}^{\prime} z_{\ell}^{\prime}$ is trivial. Hence $u \approx v$ is trivial.

This completes the proof.

Proposition 8. $\operatorname{HSP}\left(S_{55}\right)$ is the ai-semiring variety determined by the identities

$$
\begin{gathered}
x y \approx y x, \\
x y \approx x^{2} y, \\
x y \approx x y+x y z, \\
x^{2} \approx x^{2}+x .
\end{gathered}
$$

P r o o f. It is routine to check that $S_{55}$ satisfies (1.26)-(1.29). In the remainder it suffices to show that if $S_{55}$ satisfies $u \approx u+q$, where $u=u_{1}+u_{2}+\cdots+u_{m}, u_{i}, q \in X^{+}, i \in \underline{m}$, then (1.26)-(1.29) and the identities determining AI imply the identity $u \approx u+q$. Choose $Z=\left(\bigcup_{i \in \underline{m}} c\left(u_{i}\right)\right) \backslash c(q)$. By [21, Lemma 2.11] we have that $T_{2}$ satisfies $D_{Z}(u) \approx D_{Z}(u)+q$, where $D_{Z}(u)$ denotes the sum of terms $u_{i}$ for which $c\left(u_{i}\right) \subseteq c(q)$. We may assume that $D_{Z}(u)=u_{1}+u_{2}+\cdots+u_{k}$. The following two cases are necessary.

- $\ell(q)=1$. Then (1.27) and (1.29) implies $u \approx u+q$.

- $\ell(q) \geq 2$. Then there exists $u_{i}$ with $c\left(u_{i}\right) \subseteq c(q)$ such that $\ell\left(u_{i}\right) \geq 2$. Further, by (1.26)-(1.28) we have

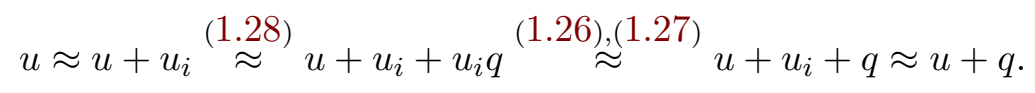

This completes the proof.

Proposition 9. $\mathbf{H S P}\left(S_{57}\right)$ is the ai-semiring variety determined by the identities

$$
\begin{gathered}
x y z \approx y x z, \\
x^{2} y \approx x y, \\
x+y z \approx y x+y z, \\
x^{2}+x y \approx x y .
\end{gathered}
$$


P r o o f. An AI-term is said to be in canonical form if it is equal to $x_{1}+\cdots+x_{m}, x^{2}$ or $x_{1} \cdots x_{m}\left(y_{1}+\cdots+y_{n}\right)$, where $\left\{x_{i} \mid i \in \underline{m}\right\} \bigcap\left\{y_{j} \mid j \in \underline{n}\right\}=\emptyset$. Let $u$ be an arbitrary AI-term. It is easy to see that there exists an AI-term $u^{\prime}$ in canonical form such that (1.30)-(1.33) imply $u \approx u^{\prime}$.

It is routine to check that $S_{57}$ satisfies (1.30)-(1.33). In the remainder it is enough to show that if $S_{57}$ satisfies $u \approx v$ where $u$ and $v$ are AI-terms in canonical form, then (1.30)-(1.33) and the identities determining AI imply $u \approx v$. Notice that both $M_{2}$ and $T_{2}$ can be embedded into $S_{57}$. We consider the following nontrivial case that $u=x_{1} \cdots x_{m}\left(y_{1}+\cdots+y_{n}\right), v=z_{1} \cdots z_{k}\left(t_{1}+\cdots+t_{\ell}\right)$. For a fixed $x_{i}$, suppose that it is not equal to $z_{j}$ for every $j \in \underline{k}$. Since $M_{2}$ can be embedded into $S_{57}$, it follows that $x_{i}$ must be equal to some $t_{j}$. Choose $x_{i}$ to 1 and every other variable to 2 . We have that $3=1$, a contradiction. Thus $x_{1} \cdots x_{m} \approx z_{1} \cdots z_{k}$ is trivial. Choose $x_{i}$ to 2 for every $i \in \underline{m}$. Then $S_{57}$ satisfies $y_{1}+\cdots+y_{n} \approx t_{1}+\cdots+t_{\ell}$. Thus $y_{1}+\cdots+y_{n} \approx t_{1}+\cdots+t_{\ell}$ is trivial and so is $u \approx v$.

Proposition 10. $\mathbf{H S P}\left(S_{58}\right)$ is the ai-semiring variety determined by the identities

$$
\begin{gathered}
x y \approx x^{2}, \\
x^{2} \approx x+x^{2} .
\end{gathered}
$$

P r o o f. An AI-term is said to be in canonical form if it is equal to $x_{1}+\cdots+x_{m}, y_{1}^{2}+\cdots+y_{n}^{2}$ or $x_{1}+\cdots+x_{m}+y_{1}^{2}+\cdots+y_{n}^{2}$, where $\left\{x_{i} \mid i \in \underline{m}\right\} \bigcap\left\{y_{j} \mid j \in \underline{n}\right\}=\emptyset$. Let $u$ be an arbitrary AI-term. It is easy to see that there exists an AI-term $u^{\prime}$ in canonical form such that (1.34) and (1.35) imply $u \approx u^{\prime}$.

It is routine to check that $S_{58}$ satisfies (1.34) and (1.35). In the remainder it is enough to show that if $S_{58}$ satisfies $u \approx v$, where $u$ and $v$ are AI-terms in canonical form, then (1.34), (1.35) and the identities determining $\mathbf{A I}$ imply $u \approx v$. Notice that $T_{2}$ can be embedded into $S_{58}$. The following two cases are necessary.

- $u=x_{1}+\cdots+x_{m}, v=y_{1}+\cdots+y_{n}$. Then $u \approx v$ is trivial.

- $u=x_{1}^{2}+\cdots+x_{m}^{2}, v=y_{1}^{2}+\cdots+y_{n}^{2}$. Since $L_{2}$ can be embedded into $S_{58}$, it follows that $u \approx v$ is trivial.

- $u=x_{1}^{2}+\cdots+x_{k}^{2}, v=y_{1}+\cdots y_{m}+z_{1}^{2}+\cdots+z_{n}^{2}$. Let $\psi: P_{f}\left(X^{+}\right) \rightarrow S_{58}$ be a semiring homomorphism such that $\psi\left(y_{i}\right)=1$ for every $i \in \underline{m}$ and $\psi(x)=2$ for every remaining variable $x$. Since $L_{2}$ can be embedded into $S_{58}$, it follows that $\psi(u)=3$ and $\psi(v)=1$, a contradiction.

- $u=x_{1}+\cdots x_{m}+y_{1}^{2}+\cdots+y_{n}^{2}, v=z_{1}+\cdots z_{k}+t_{1}^{2}+\cdots+t_{\ell}^{2}$. Since $L_{2}$ can be embedded into $S_{58}$, we have

$$
\left\{x_{i} \mid i \in \underline{m}\right\} \bigcup\left\{y_{i} \mid i \in \underline{n}\right\}=\left\{z_{j} \mid j \in \underline{k}\right\} \bigcup\left\{t_{j} \mid j \in \underline{\ell}\right\} .
$$

For a fixed $x_{i}$, suppose that it is not equal to $z_{j}$ for every $j \in \underline{k}$. Then $x_{i}$ must be equal to some $t_{j}$. Choose $x_{i}$ to 1 and every remaining variable to 2 . We have that $1=3$, a contradiction. Thus $x_{i}$ is equal to some $z_{i}$ and so $\left\{x_{i} \mid i \in \underline{m}\right\}=\left\{z_{j} \mid j \in \underline{k}\right\}$. Hence $u \approx v$ is trivial.

This completes the proof. 
Proposition 11. $\operatorname{HSP}\left(S_{59}\right)$ is the ai-semiring variety determined by the identities

$$
\begin{gathered}
x_{1} x_{2} x_{3} \approx y_{1} y_{2} y_{3}, \\
x^{3}+y=x^{3}, \\
x^{2}+y^{2} \approx x y, \\
x+x^{2} \approx x^{2} .
\end{gathered}
$$

P r o o f. An AI-term is said to be in canonical form if it is equal to $x^{3}, x_{1}+\cdots+x_{m}$, $y_{1}^{2}+\cdots+y_{n}^{2}$ or $x_{1}+\cdots+x_{m}+y_{1}^{2}+\cdots+y_{n}^{2}$, where $\left\{x_{i} \mid i \in \underline{m}\right\} \bigcap\left\{y_{j} \mid j \in \underline{n}\right\}=\emptyset$. Let $u$ be an arbitrary AI-term. It is easy to see that there exists an AI-term $u^{\prime}$ in canonical form such that (1.36)-(1.39) imply $u \approx u^{\prime}$.

It is routine to check that $S_{59}$ satisfies (1.36)-(1.39). In the remainder it is enough to show that if $S_{59}$ satisfies $u \approx v$, where $u$ and $v$ are AI-terms in canonical form, then (1.36)-(1.39) and the identities determining AI imply $u \approx v$. Notice that $T_{2}$ can be embedded into $S_{59}$. The following cases are necessary.

- $u=x_{1}+\cdots+x_{m}, v=y_{1}+\cdots+y_{n}$. It is easy to see that $u \approx v$ is trivial.

- $u=t_{1}^{3}, v=t_{2}^{3}$. Then (1.36) implies $u \approx v$.

- $u=t_{1}^{3}, v=y_{1}^{2}+\cdots+y_{n}^{2}$. Choose every variable to 2 . Then $3=1$, a contradiction. Thus $S_{59}$ does not satisfy $u \approx v$.

- $u=t_{1}^{3}, v=x_{1}+\cdots+x_{m}+y_{1}^{2}+\cdots+y_{n}^{2}$. Choose every variable to 2 . Then $3=1$, a contradiction. Thus $S_{59}$ does not satisfy $u \approx v$.

- $u=x_{1}^{2}+\cdots+x_{m}^{2}, v=y_{1}^{2}+\cdots+y_{n}^{2}$. It is easy to see that $u \approx v$ is trivial.

- $u=x_{1}^{2}+\cdots+x_{m}^{2}, v=y_{1}+\cdots+y_{k}+z_{1}^{2}+\cdots+z_{\ell}^{2}$. Consider the following two subcases.

$\diamond\left\{y_{i} \mid i \in \underline{k}\right\} \nsubseteq\left\{x_{i} \mid i \in \underline{m}\right\}$. Choose $y_{i}$ to 3 , where $y_{i} \notin\left\{x_{i} \mid i \in \underline{m}\right\}$. Choose every other variable to 2 . Then $1=3$, a contradiction.

$\diamond\left\{y_{i} \mid i \in \underline{k}\right\} \subseteq\left\{x_{i} \mid i \in \underline{m}\right\}$. Choose $y_{i}$ to 1 for every $i \in \underline{m}$ and every other variable to 2. Then $3=1$, a contradiction.

Thus $S_{59}$ does not satisfy $u \approx v$.

- $u=x_{1}+\cdots+x_{m}+y_{1}^{2}+\cdots+y_{n}^{2}, v=z_{1}+\cdots+z_{k}+s_{1}^{2}+\cdots+s_{\ell}^{2}$. Fix $x_{i}$. Suppose that $x_{i}$ is not equal to $z_{j}$ for every $j \in \underline{k}$.

$\diamond x_{i} \in\left\{s_{j} \mid j \in \underline{\ell}\right\}$. Choose $x_{i}$ to 1 and every other variable to 2 . Then $1=3$, a contradiction.

$\diamond x_{i} \notin\left\{s_{j} \mid j \in \underline{\ell}\right\}$. Choose $x_{i}$ to 3 and every other variable to 2 . Then $3=1$, a contradiction.

Thus $x_{i}$ is equal to $z_{j}$ for some $j \in \underline{k}$ and so $x_{1}+\cdots+x_{m} \approx z_{1}+\cdots+z_{k}$ is trivial. Further, $y_{1}^{2}+\cdots+y_{n}^{2} \approx s_{1}^{2}+\cdots+s_{\ell}^{2}$ holds in $S_{59}$. By the case 5 we have that this identity is trivial and so is $u \approx v$.

This complete the proof. 
Proposition 12. $\operatorname{HSP}\left(S_{60}\right)$ is the ai-semiring variety determined by the identities

$$
\begin{gathered}
x^{3} \approx x^{2}, \\
x^{2}+y^{2} \approx x y, \\
x+x^{2} \approx x^{2} .
\end{gathered}
$$

P r o o f. An AI-term is said to be in canonical form if it is equal to $x_{1}+\cdots+x_{m}, y_{1}^{2}+\cdots+y_{n}^{2}$ or $x_{1}+\cdots+x_{m}+y_{1}^{2}+\cdots+y_{n}^{2}$, where $\left\{x_{i} \mid i \in \underline{m}\right\} \bigcap\left\{y_{j} \mid j \in \underline{n}\right\}=\emptyset$. Let $u$ be an arbitrary AI-term. It is easy to see that there exists an AI-term $u^{\prime}$ in canonical form such that (1.40)-(1.42) imply $u \approx u^{\prime}$.

It is routine to check that $S_{60}$ satisfies (1.40)-(1.42). In the remainder it suffices to show that if $S_{60}$ satisfies $u \approx v$, where $u$ and $v$ are terms in canonical form, then (1.40)-(1.42) and the identities determining AI imply $u \approx v$. Notice that $T_{2}$ can be embedded into $S_{60}$. The following cases are necessary.

- $u=x_{1}+\cdots+x_{m}, v=y_{1}+\cdots+y_{n}$. It is easy to see that $u \approx v$ is trivial.

- $u=x_{1}^{2}+\cdots+x_{m}^{2}, v=y_{1}^{2}+\cdots+y_{n}^{2}$. Since $M_{2}$ can be embedded into $S_{60}$, it follows that $u \approx v$ is trivial.

- $u=x_{1}^{2}+\cdots+x_{m}^{2}, v=y_{1}+\cdots+y_{k}+z_{1}^{2}+\cdots+z_{\ell}^{2}$. Then $\left\{y_{i} \mid i \in \underline{k}\right\} \subseteq\left\{x_{i} \mid i \in \underline{m}\right\}$. Choose $y_{i}$ to 1 for every $i \in \underline{k}$ and every other variable to 2 . Then $3=1$, a contradiction. Thus $S_{60}$ does not satisfy $u \approx v$.

- $u=x_{1}+\cdots+x_{m}+y_{1}^{2}+\cdots+y_{n}^{2}, v=z_{1}+\cdots+z_{k}+s_{1}^{2}+\cdots+s_{\ell}^{2}$. Then

$$
\left\{x_{i} \mid i \in \underline{m}\right\} \bigcup\left\{y_{i} \mid i \in \underline{n}\right\}=\left\{z_{j} \mid j \in \underline{k}\right\} \bigcup\left\{s_{j} \mid j \in \underline{\ell}\right\} \text {. }
$$

Fix $x_{i}$. Suppose that $x_{i}$ is not equal to $z_{j}$ for all $j \in \underline{k}$. Choose $x_{i}$ to 1 for all $i \in \underline{k}$ and every other variable to 2 . Then $1=3$, a contradiction. Thus $x_{i}=z_{j}$ for some $j \in \underline{k}$. This implies that $u \approx v$ is trivial.

This complete the proof.

By Propositions 1-12 we immediately complete the proof of Theorem 1.

\section{Conclusion}

We have answered the finite basis problem for all ai-semirings of order three except $S_{7}$. This will lay a solid foundation for our subsequent work about ai-semiring varieties. Moreover, we conjecture that the semiring $S_{7}$ is nonfinitely based. In contrast to the rich results in the theory of semigroup varieties [26], there are still many problems to be solved in the theory of semiring varieties. In particular, it is of the interest to study the variety generated by all ai-semirings of order three.

\section{Acknowledgements}

The authors are particularly grateful to Professor Mikhail V. Volkov for his helpful suggestions contributed to this paper. The authors also thank the anonymous referees for their valuable comments and suggestions which lead to the final version of this paper. Xianzhong Zhao is supported by National Natural Science Foundation of China (11971383, 11571278). Miaomiao Ren, corresponding author, is supported by National Natural Science Foundation of China (11701449). Yong Shao is supported by Natural Science Foundation of Shaanxi Province (2020JM-425). 


\section{REFERENCES}

1. Dolinka I. A nonfintely based finite semiring. Int. J. Algebra Comput., 2007. Vol. 17, No. 8. P. $1537-1551$. DOI: $10.1142 / \mathrm{S} 0218196707004177$

2. Dolinka I. A class of inherently nonfinitely based semirings. Algebra Universalis, 2009. Vol. 60, No. 1. P. 19-35. DOI: 10.1007/s00012-008-2084-y

3. Dolinka I. The finite basis problem for endomorphism semirings of finite semilattices with zero. Algebra Universalis, 2009. Vol. 61, No. 3-4. P. 441-448. DOI: 10.1007/s00012-009-0024-0

4. Dolinka I. A remark on nonfinitely based semirings. Semigroup Forum, 2009. Vol. 78, No. 2. P. $368-373$. DOI: $10.1007 / \mathrm{s} 00233-008-9096-y$

5. Ghosh S., Pastijn F., Zhao X.Z. Varieties generated by ordered bands I. Order, 2005. Vol. 22 , No. 2. P. 109-128. DOI: 10.1007/s11083-005-9011-z

6. Kruse R. L. Identities satisfied by a finite ring. J. Algebra, 1973. Vol. 26, No. 2. P. $298-318$. DOI: $10.1016 / 0021-8693(73) 90025-2$

7. Kuřil M., Polák L. On varieties of semilattice-ordered semigroups. Semigroup Forum, 2005. Vol. 71, No. 1. P. 27-48. DOI: 10.1007/s00233-004-0176-3

8. L'vov I. V. Varieties of associative rings. I. Algebra and Logic, 1973. Vol. 12, No. 3. P. $150-167$. DOI: $10.1007 / \mathrm{BF} 02218695$

9. Lyndon R. C. Identities in two-valued calculi. Trans. Amer. Math. Soc., 1951. Vol. 71, No. 3. P. $457-457$. DOI: 10.1090/S0002-9947-1951-0044470-3

10. Lyndon R.C. Identities in finite algebras. Proc. Amer. Math. Soc., 1954. Vol. 5. P. 8-9. DOI: $10.1090 /$ S0002-9939-1954-0060482-6

11. McKenzie R. Equational bases for lattice theories. Math. Scand., 1970. Vol. 27. P. 24-38. DOI: $10.7146 /$ math.scand.a-10984

12. McKenzie R. Tarski's finite basis problem is undecidable. Int. J. Algebra Comput., 1996. Vol. 6, No. 1. P. 49-104. DOI: 10.1142/S0218196796000040

13. McKenzie R. C., Romanowska A. Varieties of -distributive bisemilattices. Contrib. Gen. Algebra, 1979. Vol. 1. P. 213-218.

14. McNulty G.F., Willard R. The Chautauqua Problem, Tarski's Finite Basis Problem, and Residual Bounds for 3-element Algebras. In progress.

15. Oates S., Powell M. B. Identical relations in finite groups. J. Algebra, 1964. Vol. 1, No. 1. P. $11-39$. DOI: 10.1016/0021-8693(64)90004-3

16. Pastijn F. Varieties generated by ordered bands II. Order, 2005. Vol. 22, No. 2. P. $129-143$. DOI: $10.1007 / \mathrm{s} 11083-005-9013-\mathrm{x}$

17. Pastijn F., Zhao X. Z. Varieties of idempotent semirings with commutative addition. Algebra Universalis, 2005. Vol. 54, No. 3. P. 301-321. DOI: 10.1007/s00012-005-1947-8

18. Perkins P. Bases for equational theories of semigroups. J. Algebra, 1969. Vol. 11, No. 2. P. $298-314$. DOI: 10.1016/0021-8693(69)90058-1

19. Ren M. M., Zhao X. Z. The varieties of semilattice-ordered semigroups satisfying $x^{3} \approx x$ and $x y \approx y x$. Period. Math. Hungar., 2016. Vol. 72, No. 2. P. 158-170. DOI: 10.1007/s10998-016-0116-5

20. Ren M. M., Zhao X.Z., Shao Y. The lattice of ai-semiring varieties satisfying $x^{n} \approx x$ and $x y \approx y x$. Semigroup Forum, 2020. Vol. 100, No. 2. P. 542-567. DOI: 10.1007/s00233-020-10092-8

21. Ren M. M., Zhao X. Z., Wang A. F. On the varieties of ai-semirings satisfying $x^{3} \approx x$. Algebra Universalis, 2017. Vol. 77, No. 4. P. 395-408. DOI: 10.1007/s00012-017-0438-z

22. Ren M. M., Zhao X. Z., Volkov M. V. The Burnside Ai-Semiring Variety Defined by $x^{n} \approx x$. Manuscript.

23. Shao Y., Ren M. M. On the varieties generated by ai-semirings of order two. Semigroup Forum, 2015. Vol. 91, No. 1. P. 171-184. DOI: 10.1007/s00233-014-9667-z

24. Tarski A. Equational logic and equational theories of algebras. Stud. Logic Found. Math., 1968. Vol. 50. P. 275-288. DOI: 10.1016/S0049-237X(08)70531-7

25. Vechtomov E. M., Petrov A. A. Multiplicatively idempotent semirings. J. Math. Sci., 2015. Vol. 206, No. 6. P. 634-653. DOI: 10.1007/s10958-015-2340-6

26. Volkov M. V. The finite basis problem for finite semigroups. Sci. Math. Jpn., 2001. Vol. 53, No. 1. 171-199. 
27. Zhao X.Z., Guo Y.Q., Shum K. P. D-subvarieties of the variety of idempotent semirings. Algebra Colloquium, 2002. Vol. 9, No. 1. P. 15-28.

28. Zhao X. Z., Shum K.P., Guo Y.Q. L-subvarieties of the variety of idempotent semirings. Algebra Universalis, 2001. Vol. 46, No. 1-2. P. 75-96. DOI: 10.1007/PL00000348 